\title{
Evaluating the Precision of Auditory Sensory Memory as an Index of Intrusion in Tinnitus
}

\author{
Doug J.K. Barrett ${ }^{1}$ and Michael Pilling ${ }^{2}$
}

${ }^{1}$ Department of Neuroscience, Psychology and Behaviour, University of Leicester, Leicester, UK.

${ }^{2}$ Department of Psychology, Oxford Brookes University, Oxford, UK.

\section{Financial Disclosures/Conflicts of interest:}

This research was part-funded by an Action on Hearing Loss summer bursary awarded to the first author.

Address correspondence to Doug J.K. Barrett, Department of Neuroscience, Psychology and Behaviour, Leicester University, Henry Wellcome Building, Lancaster Road, Leicester, LE1 9HN, UK. E-mail: djkb1@le.ac.uk Tel: +44 116229 7178. 


\begin{abstract}
Objectives: The purpose of this study was to investigate the potential of measures of auditory short-term memory (ASTM) to provide a clinical measure of intrusion in tinnitus.
\end{abstract}

Method: Response functions for six normal listeners on a delayed pitch discrimination task were contrasted in three conditions designed to manipulate attention in the presence and absence of simulated tinnitus: (i) No-Tinnitus (ii) Ignore-Tinnitus (iii) Attend-Tinnitus.

Results: Delayed pitch discrimination functions were more variable in the presence of simulated tinnitus when listeners were asked to divide attention between the primary task and the amplitude of the tinnitus tone.

Conclusions: Changes in the variability of auditory short-term memory may provide a novel means of quantifying the level of intrusion associated with the tinnitus percept during listening. 


\section{Introduction}

Sensorineural tinnitus is caused by abnormal neural activity originating in the cochlea, auditory nerve or central nervous system (Tyler et al. 2007). Its presentation is associated with hearing loss in a majority of cases (Davis \& Rafaie, 2000) and its incidence and severity increase with age (Møller, 2010). The subjective nature of tinnitus makes its severity difficult to assess: Correlations between audiometric and subjective reports of tinnitus are typically low, highlighting the role of non-auditory functions such as attention and emotion in its expression and maintenance (Erlansson, et al. 1992; Ueyama et al. 2013). Recent models implicate plastic changes in the connectivity between the auditory cortex and the limbic system, along with a maladaptive bias to attend to the tinnitus percept in the development of tinnitus-related distress (Mirz et al. 2000; Roberts et al. 2013). These changes are likely to impact negatively on hearing as perceptual and cognitive resources used to process external sounds are diverted towards the internal percept (Cuny et al., 2004; Rossiter, Stevens \& Walker, 2006).

One resource that may be particularly sensitive to competition during listening is auditory short-term memory (ASTM). In normal listeners, the precision of ASTM is inversely related to memory load (Kumar et al., 2013). Precision describes the variability of recall around the true value of the remembered stimulus, and is usually defined as the reciprocal of the standard deviation of listeners' responses during match-to-sample or continuous response tasks (Bays \& Husain, 2008; Bays, Catalao \& Husain, 2009). If changes in the precision of ASTM are an expression of competition for resources, and if problem tinnitus reflects a maladaptive bias towards the tinnitus percept, the diversion of 
resources should decrease the precision of recall for external sounds.

The current study is designed to investigate whether measures of ASTM provide a potential measure of "intrusion" in tinnitus. To assess this possibility we used a simulated tinnitus paradigm (e.g., Andersson et al. 2004; Mirz et al. 2000) to contrast normal listeners' responses on a delayed pitch discrimination task in the presence and absence of a "tinnitus-tone." In addition, we manipulated attention by asking listeners to report or ignore the amplitude of the tinnitus-tone when it was present. The former was designed to induce an attentional bias towards the tinnitus percept as is found in intrusive tinnitus, while the latter simulated the case of a listener who had habituated to the condition. If competition between sounds during encoding and maintenance modulates the precision of ASTM, recall on the delayed match-to-sample task should be more variable in the presence of simulated tinnitus; particularly when listeners are required to allocate resources to the tinnitus tone during listening.

\section{Method}

Participants

Six students from the University of Leicester were recruited to the study ( 4 females; mean age $=24.7$ years). All reported normal hearing and no neurological disorders. The School of Psychology's Research Ethics Committee approved the study.

\section{Stimuli}

Stimuli for the delayed discrimination task were pure tones. Target tones 
were centred on three reference frequencies: $668 \mathrm{~Hz}, 750 \mathrm{~Hz}$ and $842 \mathrm{~Hz}$ with an additional jitter of \pm 5 to $20 \mathrm{~Hz}$ to avoid any consolidation of targets in long-term memory. Probe tones differed from the target tone by \pm 5, 10, 20, 30 or $40 \mathrm{~Hz}$. Target and probe tones were 250 milliseconds (ms) long with $10 \mathrm{~ms}$ cosine onset and offset ramps. Tinnitus was simulated using a pure tone with a frequency of $1500 \mathrm{~Hz}$. On $50 \%$ of trials, the amplitude of the tinnitus-tone was constant. On the remaining trials, the amplitude of the tinnitus-tone was modulated by $10 \%$ using a sinusoid with a frequency of $1 \mathrm{~Hz}$. Sounds were presented binaurally at $72 \mathrm{~dB}$ SPL using headphones (HAD 200: Sennheiser Electronic Corporation).

\section{Procedure}

We used three listening conditions to compare performance in the absence and presence of simulated tinnitus: 1, No-Tinnitus 2, Ignore-Tinnitus and 3, Attend-Tinnitus. Trials started with a visual prompt to listen before the target tone was presented 0.75 seconds later. Five seconds after the offset of the target, a probe tone was presented. Participants reported whether the probe tone was higher or lower in pitch than the target using the "up" and "down" keys on a standard keyboard. On No-Tinnitus trials, probe and target tones were presented alone. On Ignore- and Attend-Tinnitus trials, the two tones were accompanied by a tinnitus tone, which started $75 \mathrm{~ms}$ before the target and finished $75 \mathrm{~ms}$ after the probe. On Ignore-Tinnitus trials, participants were instructed to focus on the pitch discrimination task. On Attend-Tinnitus trials, participants were also asked whether the tinnitus tone was constant or amplitude modulated (see Supplemental Digital Content 1). Once the response(s) had been recorded, a $250 \mathrm{~ms}$ burst of white noise signalled the start of the next 
trial.

Participants completed five blocks of 72 trials in each listening condition. Trials were blocked and the order of presentation of listening conditions was counterbalanced across participants. Before starting the experiment, training was conducted to ensure stimuli were presented at a comfortable level and participants understood the task. Stimulus presentation and data recording were controlled using custom-built software in MATLAB (Mathworks, Natick, MA, USA) with Psychophysics toolbox extensions (Brainard, 1997; Kleiner et al., 2007).

\section{Analyses}

Individual accuracy rates across target tones at each probe-target difference were transformed to the proportion of correct "higher" responses. Response distributions were fitted with a Cumulative Gaussian psychometric function:

$$
p(x, \mu, \sigma)=\frac{1}{2} \cdot \operatorname{erf}\left(\frac{x-\mu}{\sigma \sqrt{2}}\right)
$$

Where $p$ is the probability of making a "higher" response, $x$ is the probe-target difference, $\mu$ is the point of subjective equivalence (PSE), $\sigma$ is the standard deviation of the normalised Gaussian function, and erf is the Gaussian error function (see Supplemental Digital Content 2). The PSE indexes the point on the psychometric function at which higher and lower pitch responses are equally probable and $\sigma$ the increase in the probe compared to the target frequency required for the listener to respond "higher" $84 \%$ of the time. Individual $\mu$ and $\sigma$ parameters were estimated using a maximum likelihood procedure (Myung, 
2003) for each listening condition and compared across participants using nonparametric tests.

\section{Results}

** Figure $1^{* *}$

** Table $1^{* *}$

Table 1 reveals a monotonic increase in mean $\sigma$ estimates in the NoTinnitus, Ignore-Tinnitus and Attend- Tinnitus conditions. This effect is evident in Figure 1, where individual slopes for the Ignore- and Attend-Tinnitus functions are generally shallower than those in the No-Tinnitus condition. In the Attend-tinnitus condition, participants correctly identified the modulation of the tinnitus tone on an average of $73 \%$ of trials $[t(5)=14.17, p<0.001]$, indicating an ability to attend to the tinnitus as well as the target and probe tones on each trial. A Related Samples Friedman's test yielded a significant effect of listening condition on $\sigma\left[\chi^{2}(2)=7.00, p=0.030\right.$ : Mean ranks; No-Tinnitus $=1.17$, IgnoreTinnitus $=2.17$, Attend-Tinnitus $=2.67]$. Post hoc Wilcoxon's signed-ranks tests revealed a significant increase in $\sigma$ in the Attend- compared to the No-Tinnitus condition $[Z=-2.20, p=0.028, r=0.63]$ but no significant difference between $\sigma$ estimates in the No- and Ignore-Tinnitus conditions $[Z=-1.78, p=0.075, r=$ 0.51]. Differences in the PSE in the No-tinnitus, Ignore-tinnitus and Attendtinnitus were not statistically significant $\left[\chi^{2}(2)=4.00, p=0.135\right]$. The data, therefore, reveal a significant increase in the variability of delayed pitch discrimination responses when listeners were required to attend the amplitude 
of a concurrent tinnitus tone.

\section{Discussion}

We investigated whether changes in the precision of ASTM provide a potential index of intrusion in tinnitus. The results reveal an increase in the variability of normal listeners' delayed pitch discrimination responses in the presence of simulated tinnitus. Mean estimates of $\sigma$ increased monotonically from the Non-Tinnitus to the Ignore- and Attend-Tinnitus conditions, with differences between the former and latter reaching statistical significance in the Attend-tinnitus condition only. At the individual level, $\sigma$ estimates were higher in the Attend- than the No-tinnitus condition across all participants. Importantly, this increase is unlikely to reflect low-level masking, as the simulated tinnitus was separated from the probe and target tones by a large frequency difference (Moore, 1995). Increases in $\sigma$ estimates were also observed in the Ignorecompared to the No-tinnitus condition, although the increase did not reach significance level. Both contrasts revealed considerable variability in the effect of simulated tinnitus on the precision of recall across individuals. Although this reduced the power to detect differences between conditions, it also highlights differences in listeners' susceptibility to interference in the Ignore- and AttendTinnitus conditions. In clinical tinnitus, the same variability may provide an individual measure of interference from the internal percept in different listening conditions.

The results above suggest ASTM measures have the potential to provide a specific index of competition between external sounds and the tinnitus percept. 
In clinical tinnitus, quantifying intrusion in the absence of a No-Tinnitus baseline requires the comparison of response functions for different individuals. Monte Carlo sampling techniques provide a means of generating confidence intervals for individual parameter estimates (Crawford \& Garthwaite, 2007), which could be compared with normative data from appropriate controls, such as agematched listeners with different levels of hearing loss and tinnitus severity (Adjamian et al. 2014). An alternative would be to contrast response distributions for immediate and delayed pitch discrimination tasks, when targetprobe differences in the former are titrated to equate individual differences in the absence of a retention period.

The current results indicate that changes in the variability of ASTM provide a quantitative measure of intrusion from simulated tinnitus in listeners with normal hearing. Orienting attention towards the tinnitus tone produced a systematic increase in the variability of listeners' responses on a delayed pitch discrimination response task. Further work is required to determine how sensitive the same measure is to attentional biases in clinical tinnitus. Nevertheless, our results suggest differences in ASTM between individuals with tinnitus and appropriate controls may provide an objective measure of competition between external sounds and the internal percept during listening and recall. In addition, simulation studies may provide a useful tool for investigating the perceptual consequences of tinnitus on hearing, and provide further insights into the way attention and ASTM contribute to its maintenance and severity. 


\section{Acknowledgements}

We would like to thank Aleksander Nitka and Abbie Gray for assistance with data collection and Action on Hearing Loss for funding a Summer Studentship to support the project. We would also like to thank three anonymous reviewers for their constructive comments on a previous version of the manuscript.

\section{Author Contribution Statement}

Both authors contributed equally to the study design, data analyses and preparation of the manuscript. DB ran the experiment at the University of Leicester.

\section{References}

Adjamian, P., Hall, D. A., Palmer, A. R., et al. (2014). Neuroanatomical abnormalities in chronic tinnitus in the human brain. Neurosci Biobehav Rev, 45, 119-133.

Andersson, G., Khakpoor, A., Lyttkens, L. (2002). Masking of tinnitus and mental activity. Clin Otolaryngol, 27(4), 270-274.

Bays, P. M., Husain, M. (2008). Dynamic shifts of limited working memory resources in human vision. Science, 321, 851-854.

Bays, P. M., Catalao, R. F. G., Husain, M. (2009). The precision of visual working memory is set by allocation of a shared resource. J Vis, 9(10), 111.

Brainard, D.H. (1997). The Psychophysics Toolbox. Spat Vis, 10, 433-436. 
Crawford, J.R., Garthwaite, P.H. (2007). Comparison of a single case to a control or normative sample in neuropsychology: Development of a Bayesian approach. Cogn Neuropsychol, 24(4), 343-372.

Cuny, C., Noreña, A., El Massioui, F., et al. (2004). Reduced attention shift in response to auditory changes in subjects with tinnitus. Audiol. Neurootol, 9, 294-302.

Davis, A., Rafaie, E.A., (2000). Epidemiology of Tinnitus. In: Tyler, R.S. (Ed.), Tinnitus Handbook (pp. 1-24). San Diego, CA: Singular.

Erlansson, S.I., Haqllberg L.R.M., Axelsson A. (1992). Psychological and audiological correlates of perceived tinnitus severity. Int J Audiol, 31, 168-179.

Kleiner, M., Brainard, D., Pelli, D. (2007). What's new in Psychtoolbox - 3? Perception, 36(14), 1.

Kumar, S., Joseph, S., Pearson, B., et al. (2013). Resource allocation and prioritization in auditory working memory. Cogn Neurosci, 4(1), 12-20.

Mirz, F., Gjedde, A., Sødkide-Jrgensen, H., et al. (2000). Functional brain imaging of tinnitus-like perception induced by aversive auditory stimuli. Neuroreport ,11, 633-637.

Møller, A.R., 2007. Tinnitus: presence and future. Prog Brain Res, 166, 3-16.

Myung, I. J. (2003). Tutorial on maximum likelihood estimation. J Math Psychol, 47, 90-100.

Moore, B.C.J. (1995). Frequency analysis and making. In B.C.J. Moore (Ed.) Hearing (pp. 161-205). Orlando, FL: Academic Press.

Roberts, L.E., Husain, F.T., Egermont, J.J. (2013). Role of attention in the generation and modulation of tinnitus. Neurosci Biobehav Rev, 37(8): 
1754-1773.

Rossiter, S., Stevens, C., Walker, G. (2006). Tinnitus and its effect on working memory and attention. J Speech, Lang Hear Res, 49, 150-160.

Tyler, R.S., Oleson, J., Noble, W., et al. (2007). Clinical trials for tinnitus: study populations, designs, measurement variables, and data analysis. Prog Brain Res, 166: 499-509.

Ueyama, T., Donishi, T., Ukai S., et al. (2013). Brain regions responsible for tinnitus distress and loudness: A resting-state fMRI study. PLoS ONE, 8(6): e67778. 
Table 1. Individual PSE and standard deviation $(\sigma)$ estimates for best fitting cumulative Gaussian functions by listening condition

\begin{tabular}{ccccccc}
\hline & \multicolumn{2}{c}{ No-Tinnitus } & \multicolumn{2}{c}{ Ignore-Tinnitus } & \multicolumn{2}{c}{ Attend-Tinnitus } \\
\hline ID & PSE & $\sigma$ & $P S E$ & $\sigma$ & $P S E$ & $\sigma$ \\
\hline S1 & -4.97 & 98.49 & -3.02 & 104.76 & -20.13 & 167.94 \\
S2 & -1.83 & 27.96 & -0.87 & 25.42 & 5.59 & 28.50 \\
S3 & 2.31 & 37.95 & 14.65 & 46.14 & 13.05 & 42.83 \\
S4 & -0.68 & 51.44 & 14.19 & 52.99 & 8.74 & 64.31 \\
S5 & -3.62 & 20.69 & -5.85 & 31.24 & -14.75 & 38.93 \\
S6 & 3.68 & 45.36 & 6.13 & 68.71 & 2.26 & 58.51 \\
Mean & -0.85 & 46.98 & 4.20 & 54.88 & -0.87 & 66.84 \\
\hline
\end{tabular}



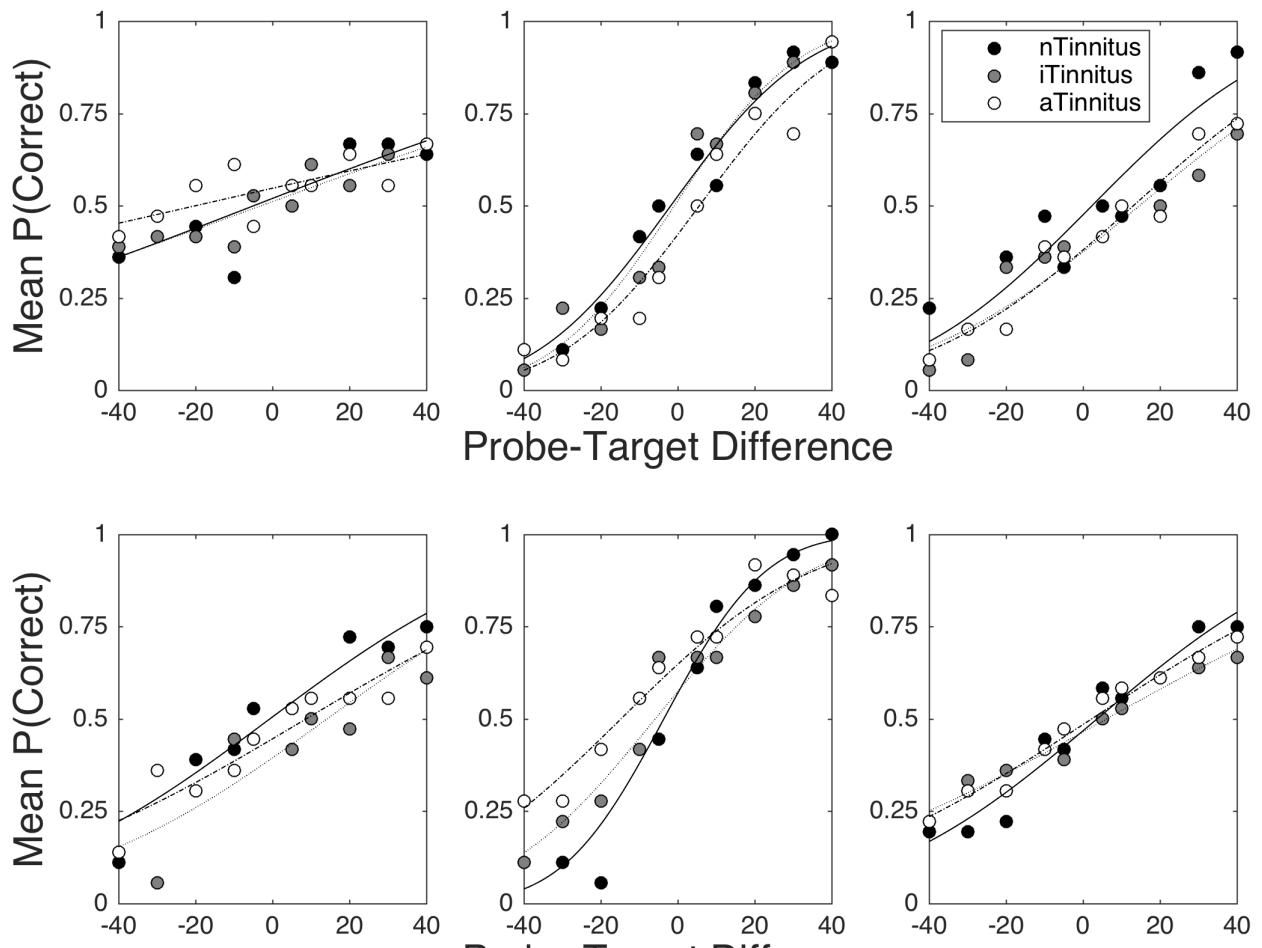

Probe-Target Difference

Figure 1. Proportion of correct higher responses and fitted psychometric functions for participants in the No-Tinnitus, Ignore-Tinnitus and AttendTinnitus conditions 


\section{Supplemental Appendix 1}

Verbal instructions were read to participants at the beginning of each experimental block. On-screen prompts were presented once the tones had finished on each trial, and remained visible until a response had been recorded.

1. No-Tinnitus Condition Verbal instructions: On each trial, you will hear two short tones. Your task is to decide whether the second tone is higher or lower in pitch than the first. Please use the appropriate key to record your response. On-screen prompts: Was the second tone lower or higher than the first (lower $=$ down arrow, higher $=$ up arrow)?

2. Ignore-Tinnitus Condition Verbal instructions: On each trial, you will hear three tones, two short and one long. Your task is to decide whether the second short tone is lower or higher in pitch than the first. Please focus on the short tones and use the appropriate key to record your response. Onscreen prompts: Was the second short tone lower or higher than the first (lower $=$ down arrow, higher $=$ up arrow)?

\section{Attend-Tinnitus Condition}

\section{Verbal instructions:}

On each trial, you will hear three tones, two short and one long. Your task is to decide whether the second short tone is lower or higher in pitch than the first, and whether the long tone is constant or modulated (steady or warbling). Please follow the on-screen prompts and use the appropriate key to record your responses.

On-screen prompts:

Was the second short tone lower or higher than the first (lower = down arrow, higher $=$ up arrow) ? Was the long tone constant or modulated (constant $=$ down arrow, modulated = up arrow)? 


\section{Supplemental Appendix 2}

Gaussian error function:

$$
\operatorname{erf}(x)=\frac{2}{\sqrt{\pi}} \int_{0}^{x} e^{-t^{2}} d t \text { for } x>0, y[0,1]
$$

Where

$$
\mathrm{t}=\frac{x}{\sqrt{2 \sigma}}
$$

